\title{
Hepatocellular carcinoma: from hepatocyte to liver cancer stem cell
}

\author{
Ioannis Karakasiliotis $^{1 *}$ and Penelope Mavromara ${ }^{2}$ \\ ${ }^{1}$ Molecular Virology, Hellenic Pasteur Institute, Athens, Greece, ${ }^{2}$ Molecular Biology and Genetics, Democritus University of \\ Thrace, Alexandroupolis, Greece
}

Keywords: HBV, HCV, hepatocellular carcinoma, liver, stem cells

OPEN ACCESS

Edited by:

Eumorphia Remboutsika,

BSRC "Alexander Fleming", Greece

Reviewed by:

Gianpaolo Papaccio,

Second University of Naples, Italy

*Correspondence: loannis Karakasiliotis, jonkost@yahoo.com

Specialty section: This article was submitted to

Craniofacial Biology, a section of the journal Frontiers in Physiology

Received: 20 April 2015 Accepted: 28 April 2015 Published: 18 May 2015

Citation: Karakasiliotis I and Mavromara P (2015) Hepatocellular carcinoma: from hepatocyte to liver cancer stem cell. Front. Physiol. 6:154. doi: 10.3389/fphys.2015.00154
Self-renewal and the potential to yield various cell types are the main attributes of stem cells and shared by the so called cancer stem cells (CSCs). CSCs consist a small portion of cells within the tumor mass that are very often resistant to chemotherapy and radiation, while being responsible for cancer relapse after treatment. The role of cancer stem cells has been recognized in cancers such as leukemia and colon cancer, whereas their role in hepatocellular carcinoma (HCC) remains to be unraveled. The vast majority of HCCs $(\sim 80 \%)$ are related to Hepatitis virus B (HBV) and $\mathrm{C}(\mathrm{HCV})$ persistent infection implicating parallel pathways of intrinsic cell transformation and chronic infection-inflammation.

CSCs reside among other more differentiated cancer cells, are resistant to apoptosis and their self-renewal is based on transcription factors similar to those of adult and embryonic stem cells such as Oct4, Nanog and Klf-4. Although their origin and relationship with the tumor initiating cell is still debatable it is rather established that they sustain tumor growth, produce differentiated progenies that organize a hierarchical cell system and induce eventually tumor's metastatic potential. Recently, it has been suggested that persistent oncogenic viruses such as HBV and HCV trigger the formation of CSCs that are possibly responsible for the high rates of recurrence and mortality that characterize HCC.

Both HBV and HCV although very different in their genome organization and life cycle they result often in persistent infection that leads eventually in 1-5\% of infected individuals to HCC through liver fibrosis and cirrhosis. HBV replicates its DNA in cell nucleus and remains episomally in the form of minichromosome. HBV DNA fragments are often integrated in host genome. HCV on the other hand is a positive strand RNA virus with cytoplasmic replication organized in virus induced vesicles. HBx protein of HBV and HCV core, NS3, and NS5A proteins are modulators of cellular pathways that lead to cell transformation. HBV and HCV chronic infection induce accumulative liver damage followed by an increasing liver regeneration that is favored by both viruses. Indeed, hepatic progenitor cells increase their numbers in the liver of HCV patients as the disease advances to cirrhosis (Tsamandas et al., 2006), while liver CD133+ (a stemness surface marker) CSCs correlated with early recurrence and poor prognosis among HBV related HCC patients (Ma et al., 2010). During cirrhosis, HBV and HCV proteins support the survival and sustain the growth of infected cells within a mayhem of inflammatory-mediated cell death.

Replication of HCV in Huh7.5 hepatoma cells in the form of a subgenomic replicon, which bears only the non-structural proteins of HCV NS3-5B, showed marked induction of liver stem cell markers such as Lgr5, cytokeratin-19 (CK-19) doublecortin and CaM kinase-like-1 (DCAMKL1) and CD133 in conjunction with liver progenitor cell makers such as $\alpha$-fetoprotein (AFP). Remarkably, curing the cells by using Interferon 2a reversed the phenomenon, while xenograftderived tumors of these cells in mice encompassed cells with liver progenitor and CSC traits (Ali et al., 2011). Biopsies from HCV-linked tumors supported the presence of DAMKL-1+ cells, whose abundance increased with the disease stage (Ali et al., 2011). Concurrently, expression of HBx protein of HBV in HepG2 hepatoma cells greatly enhanced the abundance of the pluripotency transcription factors such as Oct-4, Nanog, and Klf-4, as well as the "stemness"-associated 
markers EpCAM and $\beta$-catenin (Arzumanyan et al., 2011). Biopsies from HBV infected patients recapitulated the overexpression of EpCAM, while Oct-4 and Nanog expressing cells were detected within the tumors (Arzumanyan et al., 2011). Recently, Nanog was shown to be induced by HCV core protein through activation of STAT3. HCV core facilitated cell cycle progression in a Nanog-dependent manner (Zhou et al., 2014). Interestingly, Nanog was upregulated in the liver of NS5A expressing mice, while the effect was maximized during alcohol feeding in a TLR4-dependent manner (Machida et al., 2009).

The induction of stemness by HCV structural and nonstructural proteins through parallel pathways possibly underscores its importance in virus proliferation and persistence. Huh7.5 cells support HCV replication, in contrast to their predecessors Huh7, to a great extent due to the constitutive activation of sonic hedgehog pathway (Choi et al., 2011), which is known to be critical for liver regeneration and the control of CSC self-renewal. Although one cannot exclude the contribution of innate immunity and mir-122 in cell permissiveness to $\mathrm{HCV}$. A more direct effect of stemness-related factors in HCV replication was observed by siRNA knockdown of DCAMKL-1 which correlated well with the decline in HCV RNA abundance (Ali et al., 2011).

Conditions within the microinviroment of the infected cells could also play an important role in the programming of liver cells toward CSCs and the divergence of the multiple pathways induced by $\mathrm{HCV}$ and $\mathrm{HBV}$ toward epithelial to mesenchymal transition (EMT) or stemness. Such condition, which is often present in inflamed and malignant areas of the liver, is hypoxia. Accumulated evidence from various cancer types strongly support the hypothesis that hypoxia sustains the self-renewal characteristics of a portion of cancer cells in hypoxic niches mainly due to the upregulation of Oct4, NANOG, SOX2, Klf4, and c-myc (Mathieu et al., 2011; Muz et al., 2014). HBV and HCV modulate hypoxic pathways to adapt cells in hypoxic conditions conferring EMT characteristics (Wilson et al., 2011; Kim, 2014). Remarkably, hypoxic conditions induced HCV replication in Huh7.5 cells which correlated with activation of anaerobic cell energy production and cell proliferation (Vassilaki et al., 2013). Thus, interplay between the induction of stemness conditions through hypoxia and activation of cell energy status by HCV and through that enhancement of HCV replication may lead to a boost of both HCV spread and tumorigenesis in chronically infected individuals.

A less evaluated parameter in HCV and HBV HCC are the extrinsic signals of CSC sustainment. Cytokines such as IL-8 are secreted during $\mathrm{HBV}$ infection, while they fluctuate during disease exacerbation periods (Dunn et al., 2007). HCV NS5A could also induce IL- 8 production and elevated IL- 8 levels were linked to resistance in interferon therapy (Polyak et al., 2001a,b). IL-8 is known to sustain CSC self-renewal in cancers such as breast and pancreatic cancer increasing invasiveness (Singh et al., 2013; Chen et al., 2014). Tumor associated macrophages (TAMs) are possibly part of the niche that sustains CSCs in breast cancer and HCC (Fan et al., 2014; Lu et al., 2014). Especially for HCC, TAMs sustain CSCs (EpCAM+ cells) through TGFb1 induction of EMT (Fan et al., 2014). HBx and HCV core are the key proteins in the induction of TGFb1 signaling-dependent EMT markers, while HCV core diverts TGFb1 signaling from tumor suppression to EMT induction (Battaglia et al., 2009). IL-6 is secreted by inflammatory and stromal cells during liver regeneration and it is known to support the conversion of nonCSCs to CSCs (Kim et al., 2013). In breast cancer IL-6 promoted self-renewal and hypoxia survival through activation of Notch pathway (Sansone et al., 2007). Both HCV and HBV induce IL6 production, while increased IL- 6 levels are poor prognostic marker for either treatment outcome (HCV, Guzman-Fulgencio et al., 2012) or HCC severity (HBV, Chang et al., 2015). The link between IL- 6 and stemness was furthermore supported by the high positive correlation between IL-6 levels and Oct4/Nanog expression in HBV HCC patients (Chang et al., 2015). IL-6 sustainment of HCC progenitor cell phenotype seems to apply even in chemically induced HCC as stemness-associated markers and cell proliferation are linked to autocrine IL- 6 activation ( $\mathrm{He}$ et al., 2013).

Thus, it is becoming increasingly supported that HBV and HCV infection promotes EMT and stem cell-like characteristics in the infected liver cells that enable them to adapt and proliferate in the hypoxic inflammatory environment of the cirrhotic liver paving the ground for the occurrence of HCC (Figure 1). These characteristics seem to favor the presence of the virus in the infected liver and are possibly associated with persistent viral replication. As recurrence and mortality are still hallmarks of HCC, it is becoming a central question to clarify the network of the intrinsic induction of CSC phenotype through the activation of EMT and stemness transcription factors and the extrinsic sustainment of this phenotype by inflammatory and regenerative signals. Identification of those characteristics that differentiate liver CSCs from normal adult stem cells and liver progenitors and dissection of the CSC niche microenvironment are critical

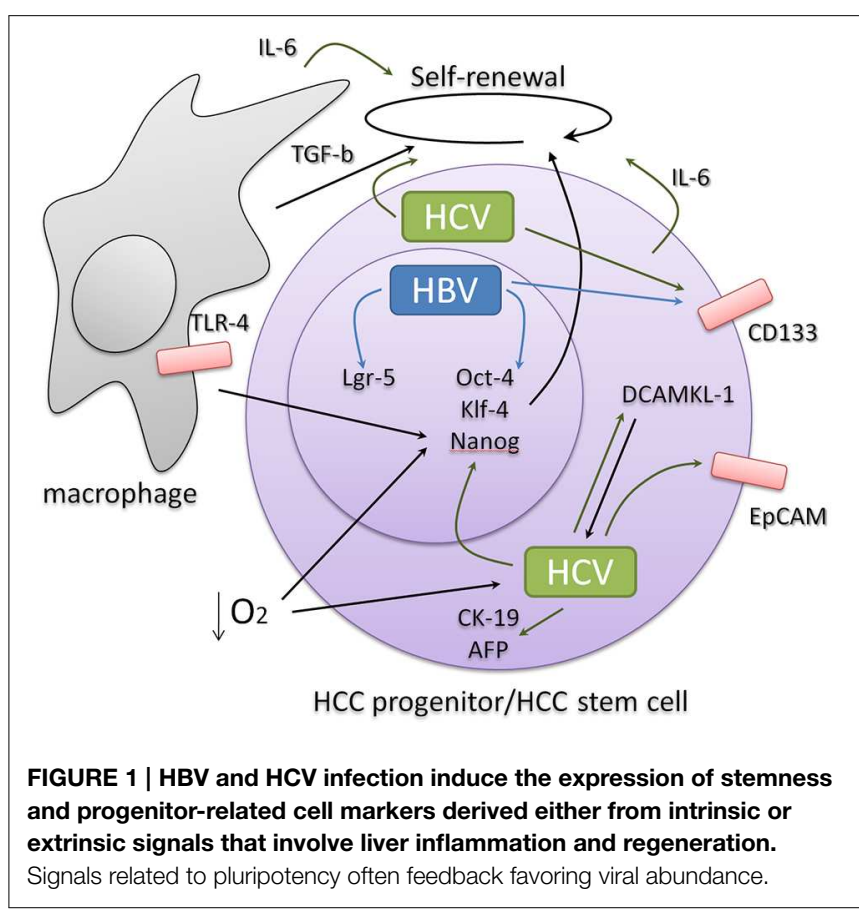


for the development of new therapies that target the root of HCC and its potential to reinitiate tumor formation after treatment.

\section{References}

Ali, N., Allam, H., May, R., Sureban, S. M., Bronze, M. S., Bader, T., et al. (2011). Hepatitis $\mathrm{C}$ virus-induced cancer stem cell-like signatures in cell culture and murine tumor xenografts. J. Virol. 85, 12292-12303. doi: 10.1128/JVI.05920-11

Arzumanyan, A., Friedman, T., Ng, I. O., Clayton, M. M., Lian, Z., and Feitelson, M. A. (2011). Does the hepatitis B antigen HBx promote the appearance of liver cancer stem cells? Cancer Res. 71, 3701-3708. doi: 10.1158/0008-5472.CAN-103951

Battaglia, S., Benzoubir, N., Nobilet, S., Charneau, P., Samuel, D., Zignego, A. L., et al. (2009). Liver cancer-derived hepatitis $C$ virus core proteins shift TGF-beta responses from tumor suppression to epithelial-mesenchymal transition. PLoS ONE 4:e4355. doi: 10.1371/journal.pone.0004355

Chang, T. S., Wu, Y. C., Chi, C. C., Su, W. C., Chang, P. J., Lee, K. F., et al. (2015). Activation of IL6/IGFIR confers poor prognosis of HBV-related hepatocellular carcinoma through induction of OCT4/NANOG expression. Clin. Cancer Res. 21, 201-210. doi: 10.1158/1078-0432.CCR-13-3274

Chen, L., Fan, J., Chen, H., Meng, Z., Chen, Z., Wang, P., et al. (2014). The IL8/CXCR1 axis is associated with cancer stem cell-like properties and correlates with clinical prognosis in human pancreatic cancer cases. Sci. Rep. 4:5911. doi: 10.1038/srep05911

Choi, S. S., Bradrick, S., Qiang, G., Mostafavi, A., Chaturvedi, G., Weinman, S. A., et al. (2011). Up-regulation of Hedgehog pathway is associated with cellular permissiveness for hepatitis C virus replication. Hepatology 54, 1580-1590. doi: 10.1002/hep. 24576

Dunn, C., Brunetto, M., Reynolds, G., Christophides, T., Kennedy, P. T., Lampertico, P., et al. (2007). Cytokines induced during chronic hepatitis B virus infection promote a pathway for NK cell-mediated liver damage. J. Exp. Med. 204, 667-680. doi: 10.1084/jem.20061287

Fan, Q. M., Jing, Y. Y., Yu, G. F., Kou, X. R., Ye, F., Gao, L., et al. (2014). Tumor-associated macrophages promote cancer stem cell-like properties via transforming growth factor-betal-induced epithelial-mesenchymal transition in hepatocellular carcinoma. Cancer Lett. 352, 160-168. doi: 10.1016/j.canlet.2014.05.008

Guzman-Fulgencio, M., Jimenez, J. L., Berenguer, J., Fernandez-Rodriguez, A., Lopez, J. C., Cosin, J., et al. (2012). Plasma IL-6 and IL-9 predict the failure of interferon-alpha plus ribavirin therapy in HIV/HCV-coinfected patients. J. Antimicrob. Chemother. 67, 1238-1245. doi: 10.1093/jac/dkr595

He, G., Dhar, D., Nakagawa, H., Font-Burgada, J., Ogata, H., Jiang, Y., et al. (2013). Identification of liver cancer progenitors whose malignant progression depends on autocrine IL-6 signaling. Cell 155, 384-396. doi: 10.1016/j.cell.2013.09.031

Kim, C. H. (2014). Hidden secret in hepatitis B viral X protein mutation and hypoxia-inducible factor-1alpha in hepatocarcinoma cancer. Hepatobiliary Surg. Nutr. 3, 115-117. doi: 10.3978/j.issn.2304-3881.2014.02.14

Kim, S. Y., Kang, J. W., Song, X., Kim, B. K., Yoo, Y. D., Kwon, Y. T., et al. (2013). Role of the IL-6-JAK1-STAT3-Oct-4 pathway in the conversion of nonstem cancer cells into cancer stem-like cells. Cell. Signal. 25, 961-969. doi: 10.1016/j.cellsig.2013.01.007

Lu, H., Clauser, K. R., Tam, W. L., Frose, J., Ye, X., Eaton, E. N., et al. (2014). A breast cancer stem cell niche supported by juxtacrine signalling from monocytes and macrophages. Nat. Cell Biol. 16, 1105-1117. doi: 10.1038/ncb3041

Ma, S., Tang, K. H., Chan, Y. P., Lee, T. K., Kwan, P. S., Castilho, A., et al. (2010). miR-130b Promotes CD133(+) liver tumor-initiating cell growth and

\section{Acknowledgments}

\author{
IK is supported by IKY SIEMENS “ARISTEIA” fellowship.
}

self-renewal via tumor protein 53-induced nuclear protein 1. Cell Stem Cell. 7, 694-707. doi: 10.1016/j.stem.2010.11.010

Machida, K., Tsukamoto, H., Mkrtchyan, H., Duan, L., Dynnyk, A., Liu, H. M., et al. (2009). Toll-like receptor 4 mediates synergism between alcohol and HCV in hepatic oncogenesis involving stem cell marker Nanog. Proc. Natl. Acad. Sci. U.S.A. 106, 1548-1553. doi: 10.1073/pnas.0807 390106

Mathieu, J., Zhang, Z., Zhou, W., Wang, A. J., Heddleston, J. M., Pinna, C. M., et al. (2011). HIF induces human embryonic stem cell markers in cancer cells. Cancer Res. 71, 4640-4652. doi: 10.1158/0008-5472.CAN-10-3320

Muz, B., de la Puente, P., Azab, F., Luderer, M., and Azab, A. K. (2014). Hypoxia promotes stem cell-like phenotype in multiple myeloma cells. Blood Cancer J. 4, e262. doi: 10.1038/bcj.2014.82

Polyak, S. J., Khabar, K. S., Paschal, D. M., Ezelle, H. J., Duverlie, G., Barber, G. N., et al. (2001a). Hepatitis C virus nonstructural 5A protein induces interleukin8 , leading to partial inhibition of the interferon-induced antiviral response. J. Virol. 75, 6095-6106. doi: 10.1128/JVI.75.13.6095-6106.2001

Polyak, S. J., Khabar, K. S., Rezeiq, M., and Gretch, D. R. (2001b). Elevated levels of interleukin-8 in serum are associated with hepatitis $\mathrm{C}$ virus infection and resistance to interferon therapy. J. Virol. 75, 6209-6211. doi: 10.1128/JVI.75.13.6209-6211.2001

Sansone, P., Storci, G., Tavolari, S., Guarnieri, T., Giovannini, C., Taffurelli, M., et al. (2007). IL-6 triggers malignant features in mammospheres from human ductal breast carcinoma and normal mammary gland. J. Clin. Invest. 117, 3988-4002. doi: 10.1172/JCI32533

Singh, J. K., Simões, B. M., Howell, S. J., Farnie, G., and Clarke, R. B. (2013). Recent advances reveal IL-8 signaling as a potential key to targeting breast cancer stem cells. Breast Cancer Res. 15:210. doi: 10.1186/bcr3436

Tsamandas, A. C., Syrokosta, I., Thomopoulos, K., Zolota, V., Dimitropoulou, D., Liava, A., et al. (2006). Potential role of hepatic progenitor cells expression in cases of chronic hepatitis $\mathrm{C}$ and their relation to response to therapy: a clinicopathologic study. Liver Int. 26, 817-826. doi: 10.1111/j.14783231.2006.01306.x

Vassilaki, N., Kalliampakou, K. I., Kotta-Loizou, I., Befani, C., Liakos, P., Simos, G., et al. (2013). Low oxygen tension enhances hepatitis $C$ virus replication. J. Virol. 87, 2935-2948. doi: 10.1128/JVI.02534-12

Wilson, G. K., Brimacombe, C. L., Rowe, I. A., Reynolds, G. M., Fletcher, N. F., Stamataki, Z., et al. (2011). A dual role for hypoxia inducible factor-1alpha in the hepatitis C virus lifecycle and hepatoma migration. J. Hepatol. 56, 803-809. doi: 10.1016/j.jhep.2011.11.018

Zhou, J. J., Chen, R. F., Deng, X. G., Zhou, Y., Ye, X., Yu, M., et al. (2014). Hepatitis $\mathrm{C}$ virus core protein regulates NANOG expression via the stat 3 pathway. FEBS Lett. 588, 566-573. doi: 10.1016/j.febslet.2013.11.041

Conflict of Interest Statement: The authors declare that the research was conducted in the absence of any commercial or financial relationships that could be construed as a potential conflict of interest.

Copyright (c) 2015 Karakasiliotis and Mavromara. This is an open-access article distributed under the terms of the Creative Commons Attribution License (CC BY). The use, distribution or reproduction in other forums is permitted, provided the original author(s) or licensor are credited and that the original publication in this journal is cited, in accordance with accepted academic practice. No use, distribution or reproduction is permitted which does not comply with these terms. 